Supplementary Information for:

\title{
Microwave-assisted [3+2]-cycloadditions of azomethine ylides
}

George Bashiardes*, Imad Safir, Achmet Said-Mohamed, Francis Barbot, Joelle Laduranty.

Département de Chimie, Méthodologie et Synthèse de Biomolécules, SFA-UMR 6514, Université de Poitiers, 40 avenue du Recteur Pineau, 86022 Poitiers, France.

Email Address: george.bashiardes@univ-poitiers.fr.

\section{Compound 7b:}

(2R，3S，3aS，9bR ; 2S，3aR，9bS)-2-Ethoxycarbonyl-1-methyl-3-phenyl-4H-[1]benzopyrano[4,3$b$ ]pyrrolidine

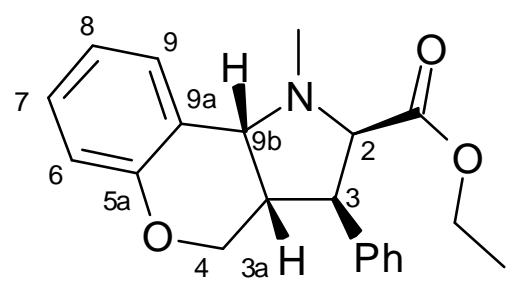

In a pyrex test tube (2x15), $O$-(3-phenyl)-allylsalicylaldehyde $\mathbf{4 b}(1 \mathrm{mmol})$, ethyl sarcosinate $\mathbf{6 a}(2 \mathrm{mmol})$ and xylene $(0.5 \mathrm{~mL})$ were submitted to microwave irradiation (CEM Discover apparatus. Settings: $\left.130^{\circ} \mathrm{C}, 100 \mathrm{~W}\right)$ during 5 minutes. After cooling, the crude reaction mixture was purified by flash column chromatography on silica gel $\left(\mathrm{CH}_{2} \mathrm{Ch}\right.$, Rf: 0.32$)$ to provide product $\mathbf{7 b}$ in $89 \%$ yield as a viscous oil.

IR $\left(\mathrm{v} \mathrm{am}^{-1}\right): 3077 \mathrm{vw}, 3056 \mathrm{vw}, 3028 \mathrm{vw}, 1606 \mathrm{vw}, 1581 \mathrm{w}, 759 \mathrm{~s}, 700 \mathrm{~s}\left(\mathrm{C}_{6} \mathrm{H}_{5}, 1,2\right.$-disub.- $\left.\mathrm{C}_{6} \mathrm{H}_{4}\right) ; 1721 \mathrm{vs}$ $(\mathrm{C}=\mathrm{O})$; 1191vs (C-O), ${ }^{1} \mathbf{H}$ NMR $\left(\mathrm{CDCl}_{3}, 300 \mathrm{MHz}\right)$ : 7.40-7.16 (m, 7H, $\left.\mathrm{H}_{7}, \mathrm{H}_{9}, \mathrm{C}_{6} \mathrm{H}_{5}\right) ; 7.04-6.92$ (m, 2H, $\left.\mathrm{H}_{8}, \mathrm{H}_{6}\right) ; 4.40\left(\mathrm{~d}, 1 \mathrm{H}, \mathrm{H}_{9 \mathrm{~b} \beta}, \mathrm{J} 9.6 \mathrm{~Hz}\right) ; 4.10-3.82\left(\mathrm{~m}, 6 \mathrm{H}, 2 \mathrm{H}_{4}, \mathrm{CH}_{2} \mathrm{O}, \mathrm{H}_{2}, \mathrm{H}_{3}\right) ; 3.50-3.42\left(\mathrm{~m}, 1 \mathrm{H}, \mathrm{H}_{3 \mathrm{a}}\right) ; 2.54$ $\left(\mathrm{s}, 3 \mathrm{H}, \mathrm{CH}_{3} \mathrm{~N}\right) ; 0.90$ (t, 3H, $\left.\mathrm{CH}_{3} \mathrm{CH}_{2}\right),{ }^{13} \mathbf{C} \mathbf{N M R}\left(\mathrm{CDCl}_{3}, 75 \mathrm{MHz}\right): 171.8(\mathrm{C}=\mathrm{O}) ; 156.8\left(\mathrm{C}_{5 \mathrm{a}}\right) ; 137.1\left(\mathrm{C}_{1^{\prime}}\right)$; $129.8\left(\mathrm{C}_{9}\right)$; 128.6, $128.5\left(\mathrm{C}_{2}, \mathrm{C}_{6^{\prime}}, \mathrm{C}_{3^{\prime}}, \mathrm{C}_{5^{\prime}}\right) ; 128.4,127.4\left(\mathrm{C}_{4}, \mathrm{C}_{7}\right) ; 126.1\left(\mathrm{C}_{9 \mathrm{a}}\right) ; 121.5,118.0\left(\mathrm{C}_{8}, \mathrm{C}_{6}\right) ; 72.3$ $\left(\mathrm{C}_{2}\right) ; 68.5\left(\mathrm{C}_{4}\right) ; 60.2\left(\mathrm{C}_{9 \mathrm{~b}}\right) ; 58.9\left(\mathrm{CH}_{2} \mathrm{O}\right) ; 48.5\left(\mathrm{C}_{3}\right) ; 43.2\left(\mathrm{C}_{3 \mathrm{a}}\right) ; 36.0\left(\mathrm{CH}_{3} \mathrm{~N}\right), 13.9\left(\mathrm{CH}_{3} \mathrm{CH}_{2}\right) ; \mathbf{M S}(\mathbf{E I})$ $\mathbf{m} / \mathbf{z}(\%): 337\left(\mathrm{M}^{+}, 3\right) ; 265\left[\left(\mathrm{M}^{+}-\mathrm{C}_{3} \mathrm{H}_{4} \mathrm{O}_{2}\right), 42\right] ; 264\left[\left(\mathrm{M}^{+}-\mathrm{C}_{3} \mathrm{H}_{5} \mathrm{O}_{2}\right), 100\right] ; 131\left[\mathrm{C}_{9} \mathrm{H}_{7} \mathrm{O}^{+}, 70\right], 91$ $\left[\mathrm{C}_{7} \mathrm{H}_{7}^{+}, 48\right] ; 77\left[\mathrm{C}_{6} \mathrm{H}_{5}^{+}, 23\right] ; 42\left[\mathrm{C}_{2} \mathrm{H}_{4} \mathrm{~N}^{+}, 45\right]$. 


\section{Compound 7d:}

(2R, 3aR, 9bR ; 2S, 3aS, 9bS)-2-Ethoxycarbonyl-1,3a-dimethyl-4H-[1]benzopyrano[4,3-b]pyrrolidine<smiles>CCOC(=O)[C@@H]1C[C@@]2(C)COc3ccccc3[C@H]2N1C</smiles>

In a pyrex test tube $(2 \times 15), O$-methallylsalicylaldehyde $\mathbf{4 d}(1 \mathrm{mmol})$, ethyl sarcosinate $\mathbf{6 a}(1 \mathrm{mmol})$ and xylene $(0.5 \mathrm{~mL})$ were submitted to microwave irradiation (Settings: $\left.130^{\circ} \mathrm{C}, 100 \mathrm{~W}\right)$ during $20 \mathrm{~min}$. After a few minutes of cooling, a further half equivalent of ethyl sarcosinate $\mathbf{6 a}(0.5 \mathrm{mmol})$ was added and the mixture was heated under irradiation again for $10 \mathrm{~min}$. After cooling and column chromatography on silica gel $\left(\mathrm{CH}_{2} \mathrm{Cl}_{2}, \mathrm{Rf}\right.$ : 0.39$)$, the product $\mathbf{7 d}$ was isolated as a colorless viscous oil in $98 \%$ yield.

IR $\left(v^{-1} \mathrm{~cm}^{-1}\right)$ : 3070w, 3035w, 1609s, 1583s, 758vs (1,2-disub. $\left.\mathrm{C}_{6} \mathrm{H}_{4}\right) ; 1729 \mathrm{vs}(\mathrm{C}=\mathrm{O}) ; 1227 \mathrm{vs}, 1180 \mathrm{vs}(\mathrm{C}-\mathrm{O})$; ${ }^{1} \mathbf{H}$ NMR $\left(\mathrm{CDCl}_{3}, 300 \mathrm{MHz}\right)$ : 7.24-7.19 (m, 2H, H, $\left.\mathrm{H}_{9}\right) ; 6.92-6.86\left(\mathrm{~m}, 2 \mathrm{H}, \mathrm{H}_{6}, \mathrm{H}_{8}\right) ; 4.22$ and 4.20 (AB of $\mathrm{ABX}_{3}$ system, $2 \mathrm{H}, \mathrm{CH}_{2} \mathrm{O}, \mathrm{J} 10.8,7.2 \mathrm{~Hz}$ ); 3.96 (A of $\mathrm{ABX}$ system, $1 \mathrm{H}, \mathrm{H}_{4 \alpha}, \mathrm{J} 10.3 \mathrm{~Hz}$ ); 3.79 (dd, 1H, $\mathrm{H}_{2 \alpha}, \mathrm{J}$ 8.1, 4.7Hz); 3.69 (B of ABX system, $\left.1 \mathrm{H}, \mathrm{H}_{4 \beta}, \mathrm{J} 10.3,1.2 \mathrm{~Hz}\right) ; 3.6\left(\mathrm{~s}, 1 \mathrm{H}, \mathrm{H}_{9 \mathrm{~b} \beta}\right) ; 2.43\left(\mathrm{~s}, 3 \mathrm{H}, \mathrm{CH}_{3} \mathrm{~N}\right) ; 1.92$ (A of $\mathrm{ABX}$ system, $1 \mathrm{H}, \mathrm{H}_{3 \alpha}, \mathrm{J} 13.6,8.1 \mathrm{~Hz}$ ); 1.88 (B of $\mathrm{ABX}$ system, $1 \mathrm{H}, \mathrm{H}_{3 \beta}, \mathrm{J} 13.6,4.8 \mathrm{~Hz}$ ); 1.31 (t, 3H, $\left.\mathrm{CH}_{3} \mathrm{CH}_{2}, \mathrm{~J} 7.2 \mathrm{~Hz}\right) ; 1.10$ (s, 3H, $\left.\mathrm{CH}_{3} \mathrm{C}\right) ;{ }^{13} \mathrm{C}$ NMR: $\left(\mathrm{CDCl}_{3}, 75 \mathrm{MHz}\right): 174.2(\mathrm{C}=\mathrm{O}) ; 154.2\left(\mathrm{C}_{5 \mathrm{a}}\right) ; 132.5$ and $132.0\left(\mathrm{C}_{9}, \mathrm{C}_{7}\right) ; 120.0\left(\mathrm{C}_{9 \mathrm{a}}\right) ; 119.8$ and $116.9\left(\mathrm{C}_{6}, \mathrm{C}_{8}\right) ; 71.9\left(\mathrm{C}_{4}\right) ; 64.7\left(\mathrm{C}_{9 \mathrm{~b}}\right) ; 63.4\left(\mathrm{C}_{2}\right) ; 60.3\left(\mathrm{CH}_{2} \mathrm{O}\right) ; 38.0$ $\left(\mathrm{C}_{3}, \mathrm{C}_{3 \mathrm{a}}\right) ; 34.8\left(\mathrm{CH}_{3} \mathrm{~N}\right) ; 20.8\left(\mathrm{CH}_{3} \mathrm{C}\right) ; 14.4\left(\mathrm{CH}_{3} \mathrm{CH}_{2}\right) ; \mathbf{M S}(\mathbf{E I}) \mathbf{m} / \mathbf{z}(\%): 275\left(\mathrm{M}^{+}, 3\right) ; 203\left[\left(\mathrm{M}^{+} \cdot \mathrm{C}_{3} \mathrm{H}_{4} \mathrm{O}_{2}\right)\right.$, 39]; $202\left[\left(\mathrm{M}^{+\cdot}-\mathrm{C}_{3} \mathrm{H}_{5} \mathrm{O}_{2}\right), 100\right] ; 145\left[\left(\mathrm{M}^{+}-\mathrm{C}_{6} \mathrm{H}_{13} \mathrm{NO}_{2}\right), 59\right], 131\left[\mathrm{C}_{9} \mathrm{H}_{7} \mathrm{O}^{+}, 29\right], 77\left[\mathrm{C}_{6} \mathrm{H}_{5}{ }^{+}, 9\right] ; 42\left[\mathrm{C}_{2} \mathrm{H}_{4} \mathrm{~N}^{+}\right.$, 57]; $29\left[\mathrm{C}_{2} \mathrm{H}_{5}^{+}, 15\right]$.

\section{Compound 8a:}

2-Carbomethoxy-N-methyl- $1 H, 4 H$-[1]benzopyrano[4,3-b]pyrrole<smiles>COC(=O)c1cc2c(n1C)-c1ccccc1OC2</smiles>

$O$-Propargylsalicylaldehyde 5a $(1.5 \mathrm{mmol})$ and methyl sarcosinate $\mathbf{6 b}(3 \mathrm{mmol})$ in xylene $0.5 \mathrm{~mL}$, were irradiated in the presence of sulphur $(7.25 \mathrm{mmol})$ at a constant temperature setting of $130^{\circ} \mathrm{C}$ with a maximal 
power output of $100 \mathrm{~W}$. After cooling, the reaction crude was filtered and the mixture was concentrated in vacuo. The residue was purified by column chromatography on silica gel (Ethyl acetate / pentane: 2/8, Rf: 0.54) to provide $8 \mathrm{a}$ in $70 \%$ yield.

IR : 3030w, 1605w, 1582w, 760m (1,2-disub.- $\left.\mathrm{C}_{6} \mathrm{H}_{4}\right) ; 1702 \mathrm{~s}(\mathrm{C}=\mathrm{O}) ; 1230 \mathrm{~s}$ and 1048w (C-O).

${ }^{1} \mathbf{H}$ NMR $\left(\mathrm{CDCl}_{3}, 300 \mathrm{MHz}\right): 7.55(\mathrm{dd}, 1 \mathrm{H}, \mathrm{H}, \mathrm{J} 7.6,2.0 \mathrm{~Hz}) ; 7.26-6.90\left(\mathrm{~m}, 3 \mathrm{H}, \mathrm{H}_{6}, \mathrm{H}_{7}\right.$ and $\left.\mathrm{H}_{8}\right) ; 6.78$ (s, $\left.1 \mathrm{H}, \mathrm{H}_{3}\right) ; 5.10\left(\mathrm{~s}, 2 \mathrm{H}, 2 \mathrm{H}_{4}\right) ; 4.18\left(\mathrm{~s}, 3 \mathrm{H}, \mathrm{CH}_{3} \mathrm{~N}\right) ; 3.82\left(\mathrm{~s}, 3 \mathrm{H}, \mathrm{CH}_{3} \mathrm{O}\right)$;

${ }^{13} \mathrm{C}$ NMR $\left(\mathrm{CDCl}_{3}, 75 \mathrm{MHz}\right): 161.6$ and $154.6\left(\mathrm{C}=\mathrm{O}, \mathrm{C}_{5 \mathrm{a}}\right) ; 131.0$ and $123.8\left(\mathrm{C}_{2}, \mathrm{C}_{9 \mathrm{~b}}\right) ; 128.5,122.2$ and $121.7\left(\mathrm{C}_{7}, \mathrm{C}_{8}, \mathrm{C}_{9}\right) ; 118.4$ and $116.2\left(\mathrm{C}_{9 \mathrm{a}}, \mathrm{C}_{3 \mathrm{a}}\right) ; 117.8\left(\mathrm{C}_{6}\right) ; 112.7\left(\mathrm{C}_{3}\right) ; 65.3\left(\mathrm{C}_{4}\right) ; 51.0\left(\mathrm{CH}_{3} \mathrm{O}\right) ; 34.8$

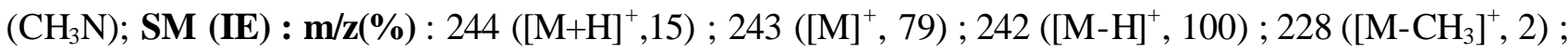
$212\left(\left[\mathrm{M}-\mathrm{OCH}_{3}\right]^{+}, 8\right) ; 184\left(\left[\mathrm{M}-\mathrm{COOCH}_{3}\right]^{+}, 12\right) ; 170\left(\left[\mathrm{C}_{11} \mathrm{H}_{8} \mathrm{NO}\right]^{+}, 2\right) ; 169\left(\left[\mathrm{C}_{11} \mathrm{H}_{7} \mathrm{NO}\right]^{+}, 13\right)$. 